\title{
Notas nomenclaturais em Stifftia J.C.Mikan (Stifftieae-Asteraceae)
}

\author{
Anna Carolina Mendonça Pereira ${ }^{1,3}$, Roberto Lourenço Esteves ${ }^{2}$, Cláudia Barbieri Ferreira Mendonça ${ }^{3}$ \\ e Vania Gonçalves-Esteves ${ }^{3,4}$
}

Recebido em 4/04/2010. Aceito em 14/07/2010

RESUMO - (Notas nomenclaturais em Stifftia J.C.Mikan (Stifftieae-Asteraceae)). Stifftia é um gênero Sul-americano, com espécies ocorrentes no Brasil e na Guiana Francesa (uma única espécie), em um total de oito táxons reconhecidos até o momento. No Brasil, o gênero é encontrado do Pará e Amapá ao Paraná, principalmente, em borda de floresta. O presente trabalho propõe novos sinônimos para Stifftia chrysantha J.C.Mikan e Stifftia parviflora (Leandro) D.Don. Palavras-chave: Asteraceae, Compositae, Stifftia, Taxonomia

ABSTRACT - (Nomenclatural notes on Stifftia J.C.Mikan (Stifftieae-Asteraceae)). Stifftia is a South-American genus, with species occurring in Brazil and French Guiana (only one species) totaling eight taxa recognized at the moment. In Brazil, the genus is present from Pará and Amapá to Paraná, mainly at forest edges. The present work suggests new synonyms for Stifftia chrysantha J.C.Mikan and Stifftia parviflora (Leandro) D.Don.

Key words: Asteraceae, Compositae, Stifftia, Taxonomy

\section{Introdução}

Stifftia é um gênero Sul-americano que ocorre no Brasil e na Guiana Francesa (uma única espécie) com um total de oito táxons reconhecidos até o momento: Stifftia cayennensis H.Rob. \& B.Kahn, Stifftia chrysantha J.C.Mikan var. chrysantha, Stifftia chrysantha var. oligantha Baker, Stifftia fruticosa (Vell.) Hind \& Semir, Stifftia hatschibachii H. Robinson, Stifftia parviflora (Leandro) D.Don, Stifftia racemosa H. Robinson, Stifftia uniflora Ducke. No Brasil, ocorre do Amazonas e Amapá ao Paraná, em bordas e no interior das matas. O gênero compreende arbustos, trepadeiras ou árvores, apresentando capítulos organizados em cimas axilares ou isolados, com pápus bem desenvolvido e colorido. Com base em estudos taxonômicos recentes dos táxons brasileiros de Stifftia (Pereira, dados inéditos), propõe-se a sinonimização de dois deles.

\section{Material e métodos}

As proposições são baseadas em revisão bibliográfica, análise de coleções históricas e recentes, incluindo tipos e fotos de tipos, disponíveis nos herbários HB, HRJ, HUEFS, MBM, NY, R, RB, RBR, SP, RFA e UEC, US, e em observações de campo realizadas no Município do Rio de Janeiro. Os acrônimos dos herbários seguem Holmgren et al. (1990). O material tipo examinado é seguido de !.

\section{Resultados e discussão}

Stifftia J.C.Mikan, Delec. Fl. Faun. Brasil. I 1, t. 1. 1820. TIPO: Stifftia chrysantha J.C.Mikan. nom. cons.
= Augusta Leandro, Denkschr. K. Baier. Akad. Wiss. 7:235. 1819. Lectótipo: (designado por Robinson 1991) Augusta grandiflora Leandro (=Stifftia chrysantha) nom. rej., non Augusta Pohl, nom. cons. (Rubiaceae).

=Aristomenia Vellozo da Conceição, Fl. Flumin. 8, t. 84 . 1825. TIPO: Aristomenia fruticosa Vellozo da Conceição. (= Stifftia chrysantha)

= Sanhilaria Leandro ex DC., Prodr. 7:26. 1838, nom. nud. pro. syn.

1. Stifftia chrysantha J.C.Mikan, Del. Fl. Faun. Bras. i. 1, t.1. 1820. TIPO: Brasil, Rio de Janeiro, J.C. Mikan s.n., s.d. (holótipo: $\mathrm{W}$; isótipo: $\mathrm{NY!)}$

= Augusta grandiflora Leandro, Denkschr. K. Baier. Akad. Wiss. 7:235. 1819. TIPO: Brasil, Rio de Janeiro, Leandro s/n. (P?, M?, fide Robinson 1991)

= Plazia brasiliensis Spreng., Neue Entd. 2, 137. 1821. TIPO: Brasil, Rio de Janeiro (herbário desconhecido).

= Aristomenia fruticosa Vellozo da Conceição. Fl. Flumin. 8: tab. 84. 1825 (1829). TIPO: Habitat silvis maritimis. Floret Nov.: Ic. fide Hind \& Semir (1998).

$=$ Stifftia chrysantha J.C.Mikan var. oligantha Baker in Martius, Fl. Bras. 6(3): 351. 1884. (= S. fruticosa (Vellozo da Conceição) D.J.N.Hind \& Semir). TIPO: habitat insula S. Sebastian: fox 89 (holótipo K).

= Stifftia chrysantha J.C.Mikan var. Alavicans Dedecca, Bragantia 13(2):23-26 (1954). TIPO: material cultivado no Instituto Agronômico de campinas, São Paulo, A. S. Lima 7306. (holótipo: SP!, isótipo: UEC) syn. nov.

Fig. 1A-E, 2A-C

\footnotetext{
1 Parte da dissertação de Mestrado da primeira Autora

2 Universidade do Estado do Rio de Janeiro, Departamento de Biologia Vegetal, Maracanã, RJ, Brasil

Universidade Federal do Rio de Janeiro, Departamento de Botânica, Museu Nacional, Rio de Janeiro, RJ, Brasil

4 Autor para correspondência: esteves.vr@gmail.com
} 
Material examinado: BRASIL. MINAS GERAIS: Monte Belo, 3/IX/1930, A. Sampaio s/n (R22391). PARANÁ: Curitiba, XII/1997, J. Carneiro 418 (MBM); Matinhos, 20/ VII/1986, R. Kummrow 2779 (MBM). RIO DE JANEIRO: Campos, III/1940, A. Sampaio 8799 (R). Itaguaí, Estrada de Itaguaí à Itacuruça, III/1950, H. Monteiro 2879 (RBR14910). Macaé: Estrada para Glicério, 23/VI/1987, T. Wendt 174 (RB); Estrada Tapera-Frade, 1/VIII/1992, C. Farney 64 (RB); Magé, Estrada de Parada Modelo para Cachoeiras de Macacu, 2/IX/2006, P. Fiaschi 3151 (RB). Nova Friburgo, Estrada Cachoeira de Macacú-Nova Friburgo, 29/IV/1972, D. Sucre 9054 (RB). Nova Iguaçu: Jaceruba, Rio São Pedro, 21/IV/1978, F. de Oliveira 406 (R); Reserva Biológica do Tinguá: 12/II/1993, M.V.L. Pereira 596 (RB); Estrada do Macuco, 17/XI/1995, M.M.T. Rosa 496 (RBR), entorno da Rebio Tinguá, RPPN do Centro de Ecologia e Cidadania do Tinguá, 20/I/2006, F.A. Sobrinho 83 (RB). Rio de Janeiro: Caminho de Belém para Rio D’Ouro, 28/VIII/1979, Franklin et Glaziou s/n (R150483); Campo Grande, Serra do Mendanha, 1/VI/1958, J. Augusto F. da Costa 40, M. Sampaio et C. Peres (R); Copacabana, VI/1897, E. Ule 4431 (R); Estrada do Rio para Serra dos Órgãos: Morro Garrafão, VIII/1974, P. Occhioni 6031 (RFA); Estada Grajaú-Jacarepaguá, 24/ VI/1958, E. Pereira 3922, Liene et al. (RB, HB, RFA); Estrada para Guaratiba, 5/VII/1984, E.F. Paciornick 38 (MBM); Horto do Jardim Botânico: III.1935, D. Constantino s/n (RFA19376); 13/III/1991, Giordano, L. C. 972 (RB); IV/1971, G. Occhioni s/n (RFA4528); VII/1975, G. Occhioni 7468 (RFA); Caminho para a Pedra do Urubu, 24/III/1992, $R$. Marquete, 537 (RB); 14/X/1992, R. Marquete, 694 (RB); 6/ II/1997, J. Bertoldo 04 (RB); 03/2006, R. C. C. Reis e equipe do projeto BBM (HB91899); Ilha do Governador: s/data, $Z$. A. trinta 1009 et E. Fromm (HB); 19/VIII/1957, G.F.J. Pabst 4312 (HB); Mata da Tijuca, VII/1942, B. Lutz 1911 (R); Morro Santa Tereza, 22/VIII., Roquete Pinto s/n (R159466); Quinta da Boa Vista, Horto Botânico, III/2005 A.C.M. Pereira s/n (R208153); Tijuca, Trapicheiro, 27/II/1944, M. Vaes $s / n$ (R41054). Seropédica, fazenda do Gerônimo, margem do Rio Guandu, 15/VIII/1951, H. Monteiro 3125 (RBR). Silva Jardim, Reserva Biológica de Poço das Antas, 16/XI/1989, R. Esteves e V. Esteves 511 (R); área FP037, 16/IV/1994, D.S. Farias 254 (RB); área P5A, 21/VI/1994, A. Piratininga 74 (RB). SÃO PAULO: Campinas, Instituto Agronômico, 19/I/1987, A. Kraprovickas Y. e L. Cristobal 40957 (MBM); Nova Europa, cultivada na fazenda Itaquerê, 28/.IV/1955, M. Kuhlmann 3674 (SP).

Distribuição geográfica: Stifftia chrysantha é encontrada nos biomas Cerrado e Mata Atlântica, em bordas de matas estacionais, matas de tabuleiro e brejos de altitude, tanto em áreas primárias como em áreas secundárias em regeneração. Ocorre, preferencialmente, na Região Sudeste, nos Estados do Rio de Janeiro, São Paulo e Minas Gerais. Segundo Baker (1884), também na Bahia. Esta é a primeira citação da espécie para os estados do Paraná e de Minas Gerais.
Comentários: Também conhecida como "flor da amizade", "diadema" e "rabo de cutia", floresce freqüentemente entre os meses de maio a agosto. Segundo Hind (1993) e Robinson (1991), a espécie é encontrada freqüentemente em cultivo nos Jardins Botânicos de diversos países à semelhança do que ocorre no Brasil. No município do Rio de Janeiro, alguns exemplares não cultivados são encontrados em platôs no Parque nacional da Tijuca.

Stifftia chrysantha var. flavicans foi estabelecida apenas com base na cor da corola, que, segundo Dedecca (1954), seria avermelhada na variedade típica e amarelada na variedade flavicans. Entretanto,observou-se que a coloração da corola como também do pápus, varia de tonalidade, de vermelho a amarelo ou ferrugíneo dependendo do estágio de maturação dos capítulos,. Assim este caráter não sustenta o estabelecimento da variedade descrita.

2. Stifftia parviflora (Leandro) D.Don., Transactions of the Linnean Society of London. 16 (2) 1833. Augusta parviflora Leandro, Denkschr. K. Baier. Akad. Wiss. 7:235 1819. TIPO: "Brazil, Near Rio de Janeiro", Leandro s.n. (P?)

= Plazia parviflora Spreng., Syst. Veg., ed.16(4):301. 1827. Fide Robinson 1991.

= Stifftia racemosa H.Rob., Systematic Botany 16(4):685692 1991. TIPO: Brazil, Minas Gerais, Capoeira, Ibirité, Município de Betin, muito frequente, 600m, 27/09/1941, Mendes Magalhães 974 (holótipo: US!) Sin. nov.

Fig. 1F, 2D-H.

Material examinado: BRASIL. ESPÍRITO SANTO: Linhares, Reserva Florestal da Cia. Vale do Rio Doce, 1/XI., S. Carvalho 038 (RBR). MINAS GERAIS: Belo Horizonte, Santana do Riacho, 7/X/1981, M.L. Kawasaki, J.R. Pirani, A. Furlan et I. Cordeiro $/ n$ (UEC27831). Cordisburgo, Fazenda do Brejão de José de Alfredo, 31/VII/1960, E.F. Heufio $s / n$ (HB32488, RB107387). Pains, Fazenda Amargoso, 9/ IX/2003, P.H.A. Melo 829 (RB). Santa Luzia, Borges, 13/ IX/1941, M. Barreto 11199 (MBM, UEC). São João Neponuceno (Lavras), VIII/1897, A. Jaguosihe s/n (R159457); RIO DE JANEIRO: Armação dos Búzios, caminho para a Praia da Ferradura, 19/VIII/1998, D. Fernandes 61 (RB, HB); São Pedro da Aldeia, Serra de Sapiatiba, 26/VIII/1997, C. Farney 3580 (RB); 24/VIII/2000, C. Farney 4137 (RB, HB); Rio de Janeiro: IX/1953, $R$. Delforge $s / n$ (RB85178); Recreio dos Bandeirantes, Jacarepaguá, 18/VIII/1959, A.P. Duarte 4977 (RB, HB); 17/VIII/1970, D. Sucre 7062 (RB); Restinga de Grumari, 29/VI/1972, J.A. de Jesus 1676 (RB); Restinga de Jacarepaguá, 18/VII/1961, A.P. Duarte 5891 (RB, UEC). SÃO PAULO: Cananéia, Parque Estadual da Ilha do Cardoso, Rio Canjoca, 21/VIII/1984, S. Romaniuc Neto \& M. Kirizawa 189 (UEC). Itapira, Beira da estrada para Lindoia, 28/VIII/1968, H.F. Leitão Filho 504 (SP, UEC); Mogi Guaçú, Fazenda Catagua, 22/IX/1992, S. Romaniuc Neto \& J. V. Godoi 1330 (SP); Piassaguéra, VIII/1923, D. Lemos 17110 (SP); São Paulo: Raiz da Serra, XII/1917, S. Schwebel s/n (SP1270). 

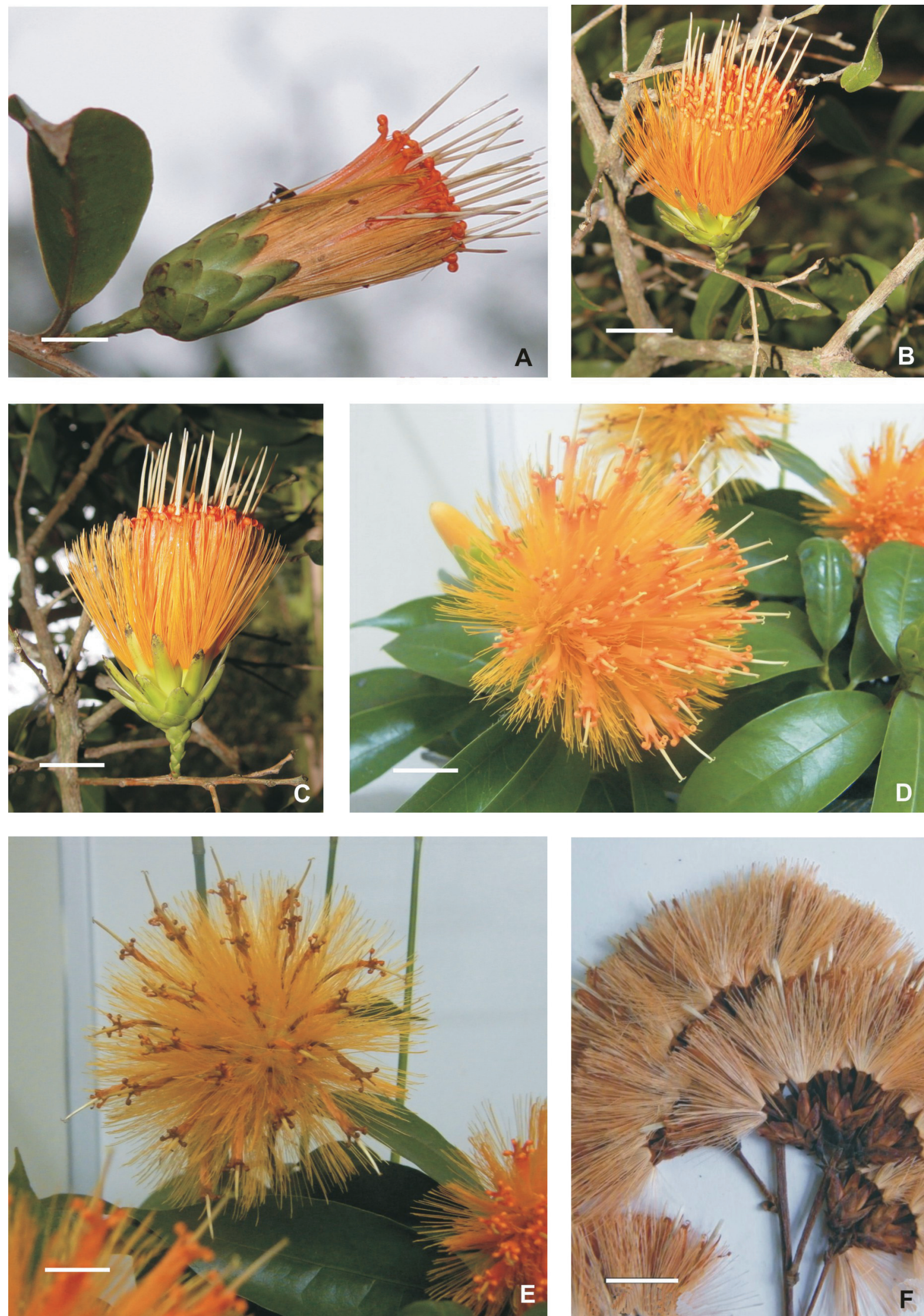

Fig. 1. Stifftia chrysantha J.C.Mikan: A-E: capítulos em vários estágios de desenvolvimento (A.C.M. Pereira s/n - R208153). S. parviflora (Leandro) D.Don: F: infrutescência evidenciando a coloração do pápus (C. Farney 4137). As escalas valem $1,0 \mathrm{~cm}$ 

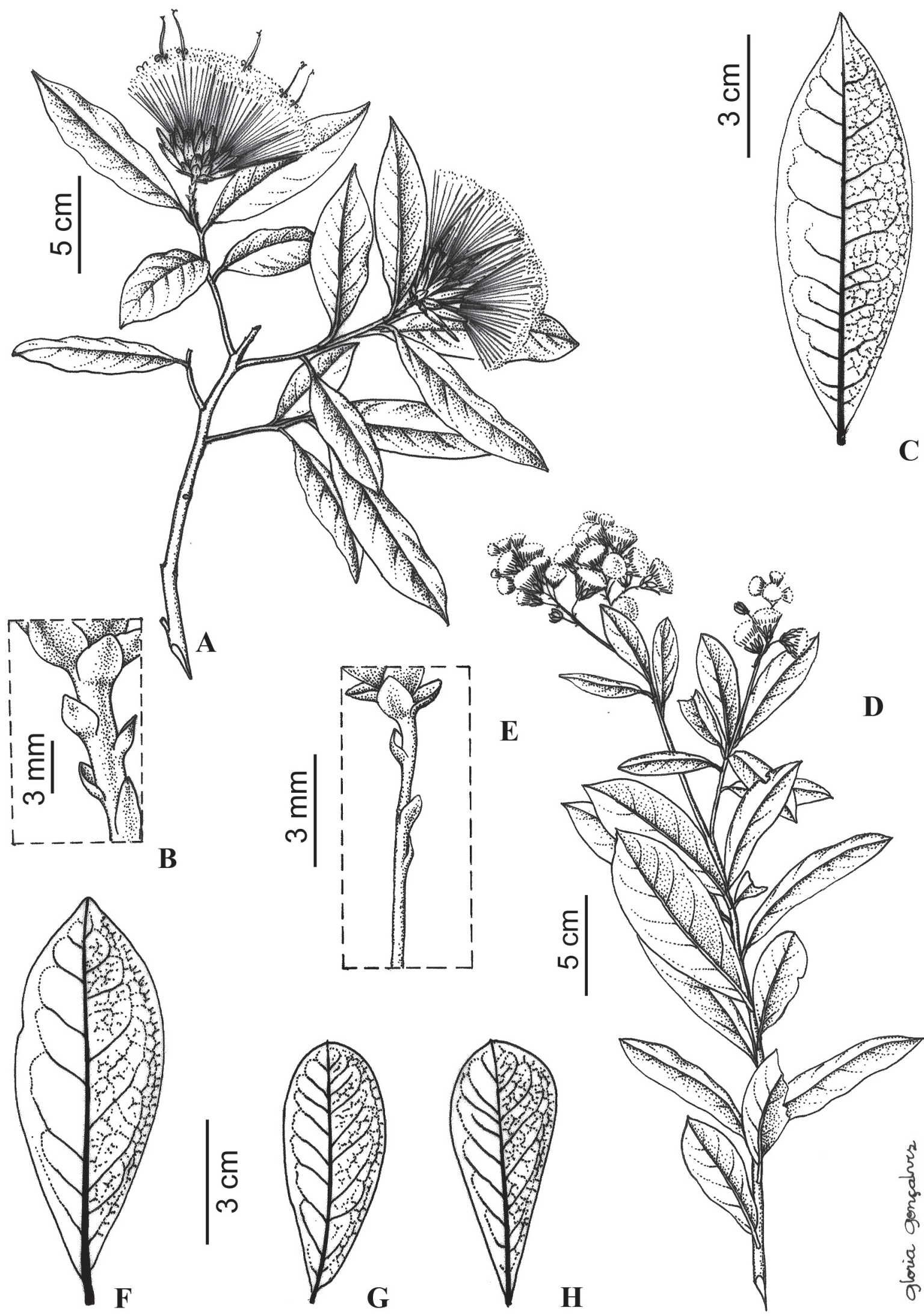

$\mathbf{E}$
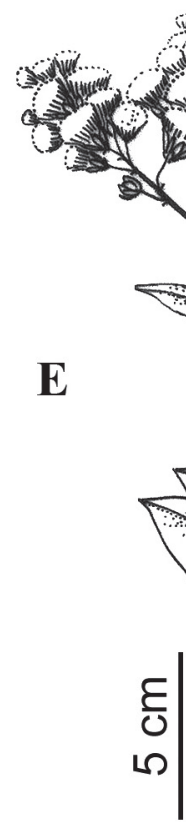

D

Fig. 2. Stifftia chrysantha J.C.Mikan: A-C: A. ramo; B. bractéolas do pedúnculo; C. lâmina foliar (A.C.M. Pereira s/n - R208153). S. parviflora (Leandro) D.Don: D-H: D. ramo; E. bractéolas; F-H. variação na forma da lâmina foliar (C. Farney 4137). 
Distribuição geografia: Stifftia parviflora encontra-se distribuída na região Sudeste (Espírito Santo, Minas Gerais, Rio de Janeiro e São Paulo), principalmente em restinga, mata de encosta e ciliar podendo ser encontrada também em mata seca, em vegetação característica da mata atlântica e do cerrado, respectivamente.

Comentários: Também conhecida como "Cambará", Stifftia parviflora apresenta poucas coletas recentes no ambiente de restinga, o que pode ser justificado pela crescente especulação imobiliária e conseqüentemente a devastação deste ambiente.

Robinson (1991) descreveu Stifftia racemosa como espécie nova para o estado de Minas Gerais com base em um único espécime. $\mathrm{O}$ autor considerou esta espécie próxima de Stifftia parviflora, diferindo-as, basicamente, pelo tipo de inflorescência, racemo na primeira e panícula na última. No material analisado, observou-se que a condição dos capítulos dispostos em pedúnculos patentes formando o conjunto um racemo curto varia muito em um mesmo espécime, tanto em comprimento como no ângulo formado entre o pedúnculo e o eixo principal da inflorescência. Por outro lado, encontraram-se, comumente, panículas apresentando entrenós muito curtos, caracterizando uma condição intermediária entre um racemo e uma panícula. Robinson (1991) também valoriza para o estabelecimento da nova espécie, a ausência de bractéolas no único exemplar examinado. A rigor, a presença dessas bractéolas é extremamente variável no conjunto do material examinado não servindo, portanto, como característica diagnóstica. Alguns exemplares com todas as características de Stifftia parviflora apresentam pedúnculos totalmente desprovidos de bractéolas ou com um número reduzido delas. Sendo os caracteres descritos por Robinson (1991) para Stifftia parviflora e Stifftia racemosa de difícil aplicação, optou-se por considerar a espécie descrita por este autor como sinônimo de Stifftia parviflora. Roque \& Pirani (1997) não aceitaram, também, S. racemosa como espécie distinta de Stifftia parviflora, embora não oficializassem a sinonímia agora proposta.

Stifftia parviflora encontra-se na categoria "provavelmente extinta", sem coleta nos últimos 30 anos para a flora de Minas Gerais. (Deliberação Conselho Estadual de Política Ambiental (COPAM), número 85, de 21 de Outubro de 1997). No entanto, a revisão nos herbários permitiu constatar coletas recentes da espécie para o Estado de Minas Gerais no ano de 2003.

\section{Agradecimentos}

Ao CNPq pela bolsa de Produtividade em Pesquisa concedida à última autora. À FAPERJ pelo auxílio à Pesquisa concedido ao Laboratório de Taxonomia de Vegetais Vasculares, UERJ e ao Laboratório de Palinologia do Museu Nacional/UFRJ

\section{Referências bibliográficas}

Baker, J.G. 1884. Stifftia in Compositae IV. Helianthoideae, Helenioideae, Anthemideae, Senecionidae, Cynaroideae, Ligulateae, Mutisiaceae. Pp.350-352. In: Von Martius, C.F.P. Flora Brasiliensis, v.6, n.3.

Copam 085/97. Lista das espécies ameaçadas de extinção da flora do Estado de Minas Gerais. Disponível em: http://www.biodiversitas.org.br/ florabr/MG-especies-ameacadas.pdf. Acesso em: novembro de 2008.

Dedecca, D.M. 1954. Uma nova variedade de Stifftia chrysantha Mikan. Bragantia 13(2): 23-26.

Holmgren, P.K.; Holmgren, N.H.; Barnett, L. 1990. Index herbariorum. Part 1: The herbaria of the world. 8 ed. New York, New York Botanical Garden.

Hind, D.J.N. 1993. Notes on the Compositae of Bahia, Brazil: I. London. Kew Bulletin 48(2): 245-277.

Robinson, H. 1991. Two new species of Stifftia with notes on relationships of the genus (Asteraceae: Mutisieae). Systematic Botany 164: 685-692.

Roque, N. \& Pirani, J.R. 1997. Flora da serra do Cipó, Minas Gerais, Compositae- Barnadesiodeae e Mutisieae. Boletim de Botânica da Universidade de São Paulo 16: 151-185. 